\title{
PRMT2 $\beta$, a C-terminal splice variant of PRMT2, inhibits the growth of breast cancer cells
}

\author{
JING ZHONG $^{1}$, YA-JUN CHEN ${ }^{1,2}$, LING CHEN ${ }^{1}$, YING-YING SHEN ${ }^{1}$, QING-HAI ZHANG ${ }^{1}$,

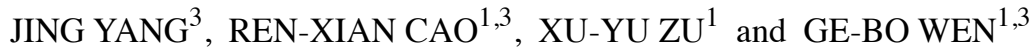 \\ ${ }^{1}$ Institute of Clinical Medicine, The First Affiliated Hospital of University of South China; \\ ${ }^{2}$ Department of Metabolism and Endocrinology, The Second Affiliated Hospital of University of South China; \\ ${ }^{3}$ Department of Metabolism and Endocrinology, The First Affiliated Hospital of University of South China, \\ Hengyang, Hunan 421001, P.R. China
}

Received January 5, 2017; Accepted June 27, 2017

DOI: $10.3892 /$ or.2017.5786

\begin{abstract}
Our previous study reported several alternative splicing variants of arginine N-methyltransferase 2 (PRMT2), which lose different exons in the C-terminals of the wild-type PRMT2 gene. Particularly, due to frame-shifting, PRMT2 $\beta$ encodes a novel amino acid sequence at the $\mathrm{C}$-terminus of the protein, the function of which is not understood. In the present study, we determined the role of PRMT2 $\beta$ in breast cancer cell proliferation, apoptosis and its effect on the Akt signaling pathway. Stable breast cancer MCF7 cell line with lentivirus-mediated PRMT2 $\beta$ overexpression was obtained after selection by puromycin for 2 weeks. The effect of lentivirus-mediated PRMT2 $\beta$ overexpression on breast cancer cellular oncogenic properties was evaluated by MTT, colony formation, cell cycle analysis and apoptosis assays in MCF7 cells. Luciferase activity assay and western blot analysis were performed to characterize the effects of PRMT2 $\beta$ on cyclin DI promoter activities and the Akt signaling pathway. Tissue microarray was performed to investigate the association of PRMT2 $\beta$ with breast cancer progression. Lentivirus-mediated PRMT $2 \beta$ overexpression suppressed the cell proliferation and colony formation of breast cancer MCF7 cells. PRMT2 $\beta$ overexpression induced cell cycle arrest and apoptosis of MCF7 cells. Furthermore, PRMT2 $\beta$ was revealed to suppress
\end{abstract}

Correspondence to: Professor Ge-Bo Wen or Dr Xu-Yu Zu, Institute of Clinical Medicine, The First Affiliated Hospital of University of South China, 69 Chuanshan Road, Hengyang, Hunan 421001, P.R. China

E-mail: wen_gb@hotmail.com

E-mail: zuxuyu0108@hotmail.com

Abbreviations: PRMT2, arginine N-methyltransferase 2; AR, androgen receptor; ER, estrogen receptor; PR, progesterone receptor; HER2, human epidermal growth factor receptor 2; AP-1, activator protein-1; Dox, doxycycline hyclate; FBS, fetal bovine serum; GFP, green fluorescent protein

Key words: PRMT2 $\beta$, antitumor, proliferation, breast cancer the transcription activity of the cyclin DI promoter, and PRMT2 $\beta$ was also found to inhibit cyclin D1 expression via the suppression of Akt/GSK-3 $\beta$ signaling in breast cancer cells. Clinically, it was revealed that PRMT2 $\beta$ expression was negatively correlated with human epidermal growth factor receptor 2 (HER2) $(\mathrm{p}=0.033)$ in breast tumors. Our results revealed that PRMT2 $\beta$, a novel splice variant of PRMT2, plays potential antitumor effect by suppressing cyclin D1 expression and inhibiting Akt signaling activity. This also opens a new avenue for treating breast cancer.

\section{Introduction}

Alternative splicing is a sophisticated and ubiquitous nuclear process in eukaryotic cells by which a single gene may yield various mRNAs, leading to products of multiple protein isoforms. In humans, more than $70 \%$ of genes are alternatively spliced $(1,2)$, which greatly increases the diversity of proteins that can be encoded by the surprisingly low number of human genes. Alternative splicing is known to play a role in the alteration of the binding properties of proteins, their enzymatic activity, their intracellular localization, or their stability and post-translational modifications $(3,4)$. Recently increasing evidence has revealed that alternative splicing plays an important role in tumorigenesis and tumor progression, and splice variants represent potentially useful targets for the development of novel therapeutic strategies $(5,6)$.

Human protein arginine N-methyltransferase 2 (PRMT2, HRMT1L1) is a protein that belongs to the arginine methyltransferase family and has weak methyltransferase activity on a histone $\mathrm{H} 4$ substrate. However, its optimal substrates have not yet been identified $(7,8)$. Subsequent studies have revealed that PRMT2 is clearly involved in a variety of cellular processes, including lung function, the inflammatory response, apoptosis promotion, and Wnt and leptin signaling regulation (9-13), suggesting that PRMT2 has diverse roles in transcriptional regulation through different mechanisms depending on its binding partners.

Our previous study demonstrated that the PRMT2 gene produces several isoforms through complex alternative splicing at the $3^{\prime}$ end of the pre-mRNA, and each has a unique C-terminal 
sequence (14). Due to the distinct gene structure characteristics, each isoform is therefore predicted to be functionally different. PRMT2 $\beta$ is the most different isoform compared with the wildtype. Sequence analysis revealed that PRMT2 $\beta$ deletes exon 7-9 in its coding sequence, and downstream frame-shifting. As a result, PRMT $2 \beta$ encodes 83 new amino acids at the C-terminus of the protein. We had previously demonstrated the negative effect of PRMT2 on breast cancer cell proliferation in vitro and in vivo. Moreover, cyclin D1 (CCND1) was found to be downregulated by PRMT2, and PRMT2 was further shown to suppress the estrogen receptor $\alpha(\mathrm{ER} \alpha)$ binding affinity to the activator protein-1 (AP-1) site in the $C C N D 1$ promoter through indirect binding with the AP-1 site, resulting in the inhibition of the CCND1 promoter activity in MCF-7 cells (15). These studies however, neglected to examine the specific functional contributions of PRMT $2 \beta$ to breast cancer progression. In the present study, we revealed that PRMT2 $\beta$ suppressed the cell proliferation and colony formation of breast cancer MCF7 cells, and induced cell cycle arrest and apoptosis of MCF7 cells. PRMT2 $\beta$ was revealed to suppress the transcription activity of the CCND1 promoter, and PRMT2 $\beta$ was also found to inhibit CCND1 expression via the suppression of Akt/GSK-3 $\beta$ signaling in breast cancer cells.

\section{Materials and methods}

Cell culture. MCF7 and 293T cells were purchased from American Type Culture Collection (ATCC) (Manassas, VA, USA), and cultured as previously described (14). Ethics approval and consent for the use of the human tissue was confirmed by the Ethics Committee of The First Affiliated Hospital of the University of South China.

Lentiviral vector construction and lentivirus infection. Recombined lentiviral vector $p G C-L V-G V 308-P R M T 2 \beta$ with the variant PRMT2 $\beta$ (FJ436411) gene (LV-Tet-on-PRMT2 $\beta$ ) and $p G C-L V-G V 308-P R M T 2$ (NM_001535) with the wildtype PRMT2 (LV-Tet-on-PRMT2) gene were constructed by the GeneChem Co. (Shanghai, China). The $p G C-L V-G V 308$ vector was used as a negative control. The packaging plasmid pHelper 1.0 and pHelper 2.0 were purchased from GeneChem Co.

We co-transfected the $p G C-L V-G V 308-P R M T 2 \beta$ or $p G C-L V-G V 308-P R M T 2$ vectors with the pHelper 1.0 and pHelper 2.0 packaging plasmid into $293 \mathrm{~T}$ cells to generate recombinant lentiviruses. Culture medium was collected $72 \mathrm{~h}$ post-transfection, and MCF7 cells were then infected with the aforementioned lentiviruses. A total of $5 \times 10^{5}$ MCF7 cells were seeded into a 6-well cell plate and further incubated for $12 \mathrm{~h}$ to reach $30 \%$ confluency, and then infected with LV-Tet-on-PRMT2 $\beta$ (PRMT2 $\beta$ overexpression group) or LV-Tet-on-PRMT2 (PRMT2 overexpression group) for $48 \mathrm{~h}$ in the presence of $8 \mu \mathrm{g} / \mathrm{ml}$ of Polybrene (Sigma-Aldrich, St. Louis, MO, USA). Stable cell lines either with the PRMT2 $\beta$ 3Flag or the PRMT2-3Flag were obtained after infection with LV-tet-on-PRMT2 $\beta$ or LV-tet-on-PRMT2 cells which were selected by puromycin for 2 weeks. Western blot analysis was performed to verify the expression of PRMT2 $\beta$-3Flag or PRMT2-3Flag induced by $5 \mu \mathrm{g} / \mathrm{ml}$ of Dox in the infected MCF7 cells.
Cell proliferation and colony formation assays. For cell proliferation assays, MCF7 cells were seeded in a 96-well plate (2,000 cells/well) and counted using an automated cell counter (Nexcelom Bioscience, Lawrence, MA, USA). For the colony formation assay, the cells were seeded in a 6-well plate (600 cells/well) and maintained for 8 days. The culture medium with or without $5 \mu \mathrm{g} / \mathrm{ml}$ of Dox was changed every 2 days. Each experiment was carried out in triplicate and performed at least twice.

Soft agar colony formation assay. The assay was performed in 6-well plates. A bottom layer of agar $(0.6 \%)$ with enriched Dulbecco's modified Eagle's medium (DMEM), [final 10\% fetal bovine serum (FBS)] was poured first. After the bottom agar solidified, MCF7 cells $\left(3.0 \times 10^{3}\right)$ were seeded in top agar $(0.3 \%)$ with enriched DMEM supplemented with $20 \%$ FBS and incubated at $37^{\circ} \mathrm{C}$ for 15 days. The culture medium with or without $5 \mu \mathrm{g} / \mathrm{ml}$ of Dox was changed twice weekly. Colonies were visualized by staining for $1 \mathrm{~h}$ with $0.005 \%$ crystal violet.

Cell cycle analysis by flow cytometry. After transfection, the MCF7 cells were plated in 6 -well plates at $2 \times 10^{5}$ cells/well. Following the designated treatments, the cells were harvested by trypsinization and washed with PBS and fixed in ice-cold $75 \%$ ethanol overnight at $-20^{\circ} \mathrm{C}$. The fixed cells were washed, and dissolved in RNAse and subsequently incubated at $37^{\circ} \mathrm{C}$ for $30 \mathrm{~min}$. Next, the cells were stained with propidium iodide (PI) for $30 \mathrm{~min}$. The DNA content of the cells $\left(1 \times 10^{4}\right.$ cells/experimental group) was determined using a BD Accuri C6 flow cytometer (BD Biosciences, Franklin Lakes, NJ, USA).

Transient transfection and luciferase activity assay. Transient gene delivery was carried out to assess the effect of PRMT2 $\beta$ and PRMT2 on the CCND1 promoter activity in 293T cells as previously described (14). A luciferase assay kit (Promega, Madison, WI, USA) was used to assess the reporter activity according to the manufacturer's instructions. Luciferase activity was normalized using a Renilla luciferase internal control.

Western blot analysis. Total cell or tissue lysates were lysed on ice for $30 \mathrm{~min}$. Soluble proteins $(20 \mu \mathrm{g})$ were probed with anti-PRMT2, anti-Flag and anti-CCND1 antibodies (1:500; Abcam, Cambridge, MA, USA) and anti-p-AKT, anti-AKT, anti-p-GSK-3 $\beta$ and anti-GSK-3 $\beta$ (1:800; Cell Signaling Technology, Danvers MA, USA). Loading variations were normalized against $\beta$-actin, which was identified by anti- $\beta$-actin monoclonal Ab (1:1,000; Abcam).

Tissue microarray and immunohistochemical analysis. The tissue microarray (BR1503b; US Biomax, Inc., Rockville, MD, USA) consisting of 138 breast malignant tumor cases, 6 normal cases and 6 benign tumor cases was utilized, and was histologically interpretable and analyzed for the correlation with clinicopathological parameters. IHC staining was performed as detailed in our previous study (14). The rabbit polyclonal PRMT2 (1:50; Aviva Systems Biology, Beijing, China) was used.

We developed affinity-purified rabbit polyclonal anti-PRMT2 $\beta$ Abs as a custom service from Boster (Wuhan, 

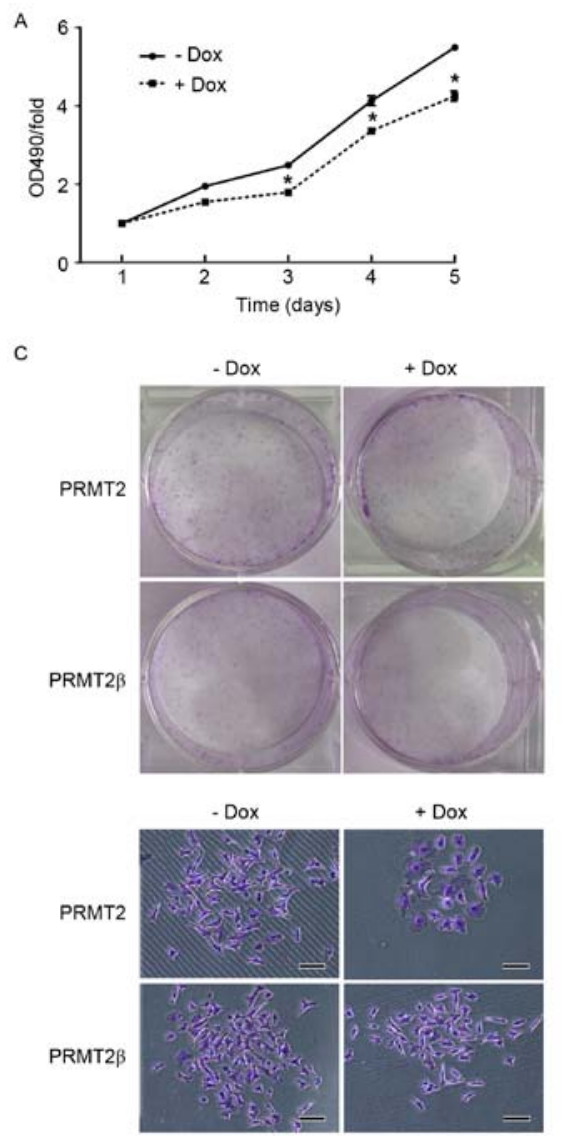

D

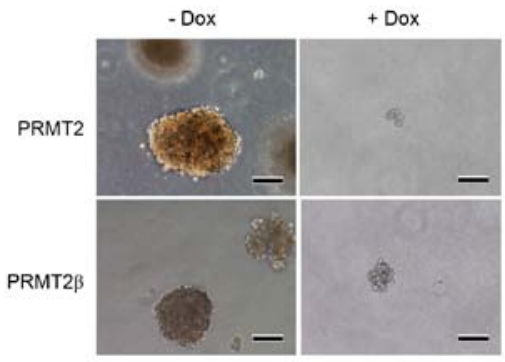

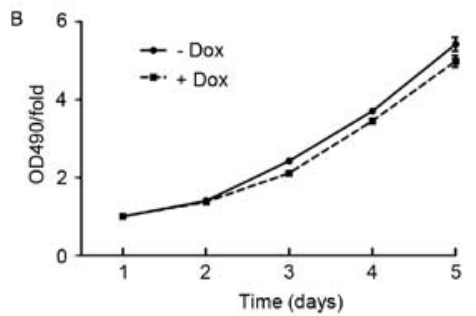
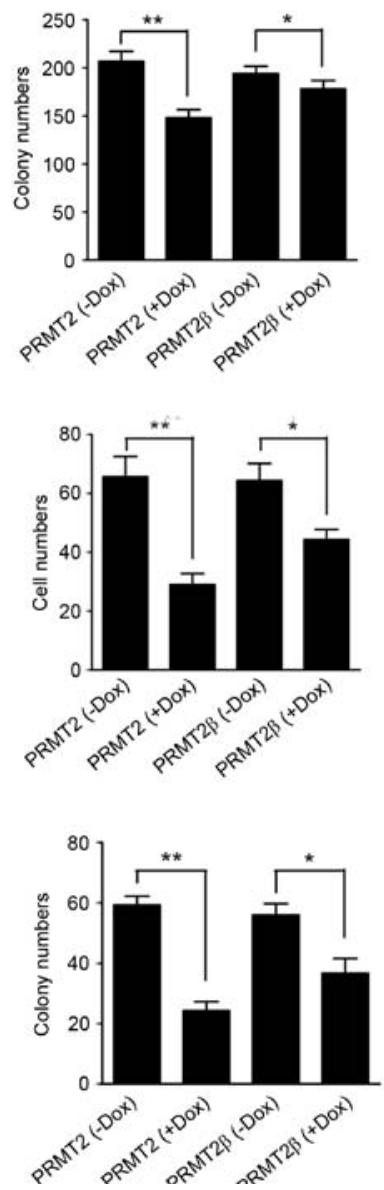

Figure 1. Lentivirus-mediated PRMT2 $\beta$ overexpression inhibits breast cancer MCF7 cell proliferation. (A and B) Growth curves of cells in each group. MTT analysis revealed that lentivirus-mediated PRMT2 and PRMT2 $\beta$ overexpression inhibited breast cancer cell proliferation $(\mathrm{n}=3)$; ${ }^{*} \mathrm{p}<0.05$. $(\mathrm{C}) \mathrm{Colony}$ formation assay revealed that lentivirus-mediated PRMT2 and PRMT2 $\beta$ overexpression significantly inhibited breast cancer cell colony formation and the cell numbers of each colony $\left(\mathrm{n}=3,{ }^{*} \mathrm{p}<0.05,{ }^{* *} \mathrm{p}<0.01\right)$. Scale bar, $50 \mu \mathrm{m}$. (D) Soft agar colony formation assay revealed that lentivirus-mediated PRMT2 and PRMT2 $\beta$ overexpression inhibited breast cancer cell soft agar colony formation $\left(\mathrm{n}=3,{ }^{*} \mathrm{p}<0.05,{ }^{* *} \mathrm{p}<0.01\right)$. Scale bar, $200 \mu \mathrm{m}$. PRMT2, arginine N-methyltransferase 2 ; Dox, doxycycline hyclate.

China). The Abs were raised against a synthetic peptide antigen corresponding to the unique C-terminal 15 aa of PRMT2 $\beta$ (HLEMTVDALFGKQCA). The specificity of the $\mathrm{Ab}$ was tested in the PRMT2 $\beta$ expression vector-transfected $293 \mathrm{~T}$ cells that do not express endogenous PRMT2 $\beta$. Immunofluorescence was used to demonstrate immunoreactive signals only in transfectants with the PRMT2 $\beta$-expressing vectors, but not in transfectants harboring an empty expression vector.

Statistical analysis. All experiments were performed using 3 replicates and the results are expressed as the mean \pm SEM or mean \pm SD. Statistical analysis was carried out using SPSS, version 13.0 (SPSS, Inc., Chicago, IL, USA). A statistical association between clinicopathological and molecular parameters was tested, using the non-parametrically two-tailed Mann-Whitney U test. p-values $<0.05$ were considered significant. Spearman's rank correlation coefficients were used to assess the correlation of PRMT2 $\beta$ and PRMT2 expression with clinicopathological parameters.

\section{Results}

PRMT2 $\beta$ suppresses the cell proliferation and colony formation of breast cancer MCF7 cells. Our previous study reported that suppression of PRMT2 expression promoted the proliferation of breast cancer cells (15). To address the cellular and molecular mechanisms of the alternatively spliced PRMT2 in breast cancer cells, a tetracycline (doxycycline hyclate, Dox)-inducible lentiviral system was 

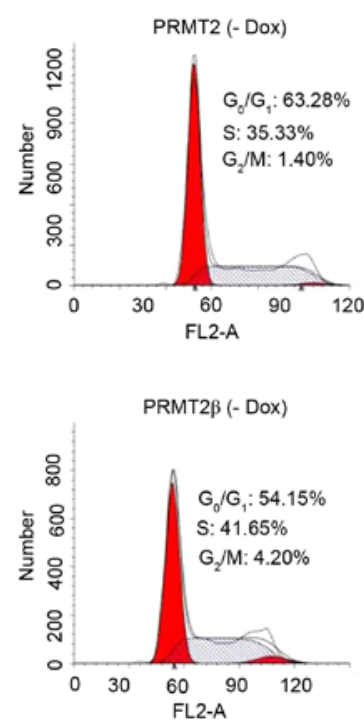

B

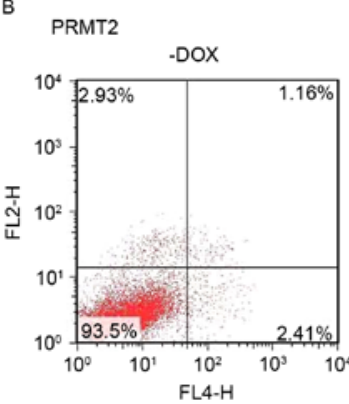

PRMT2 $\beta$

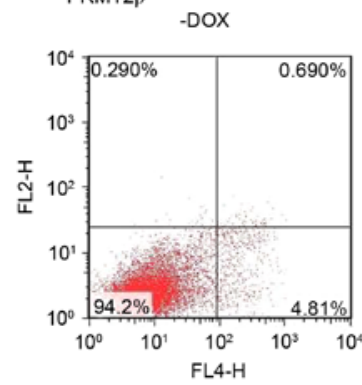

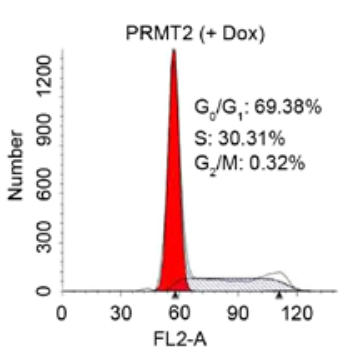
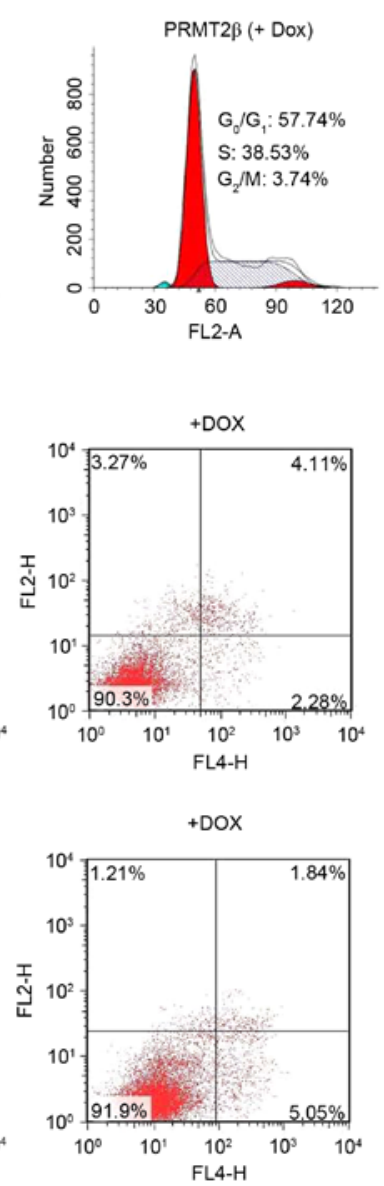
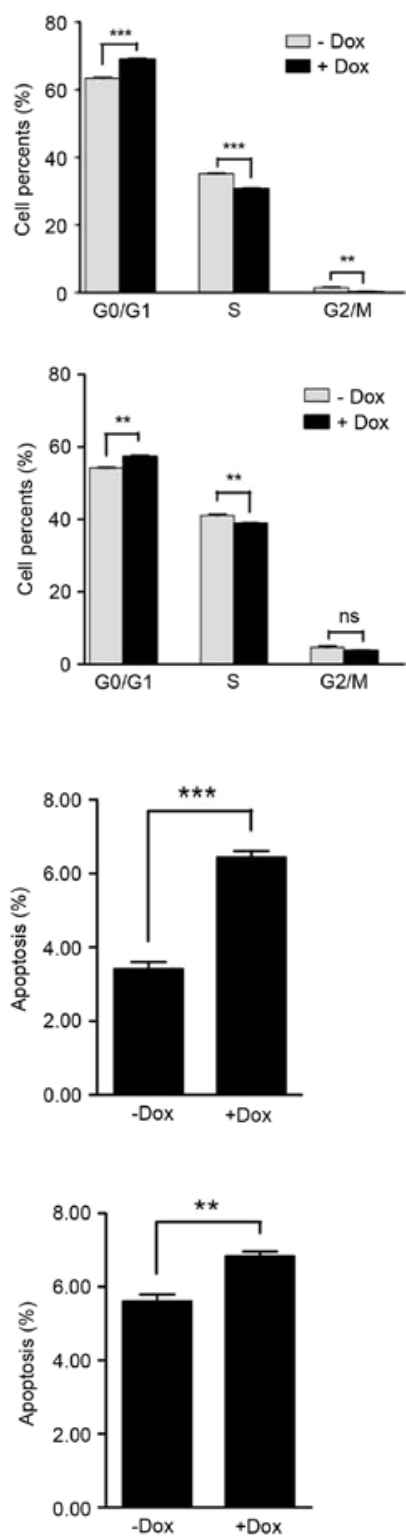

Figure 2. PRMT2 $\beta$ induces cell cycle arrest and apoptosis of MCF7 cells. (A) Detection of the proportion of breast cancer MCF7 cells in the cell cycle stages after overexpression of PRMT2 and PRMT2 $\beta$, respectively. The proportion of cells in the $G_{0}-G_{1}$ phase of the cell cycle increased and the proportion of cells in the S phase decreased in the PRMT2 and PRMT2 $\beta$ groups $\left({ }^{* *} \mathrm{p}<0.01,{ }^{* * *} \mathrm{p}<0.001\right)$. Bar heights represent the mean of the data and bars represent the SEM of the data obtained in 3 independent experiments. (B) Flow cytometric analysis of MCF7 cells in the - Dox and + Dox group. Comparison of the percentage of apoptotic cells between the - Dox and + Dox groups. PRMT2 and PRMT2 $\beta$ overexpression significantly increased the percentage of apoptotic cells when compared with - Dox group $\left({ }^{* *} \mathrm{p}<0.01,{ }^{* * *} \mathrm{p}<0.001\right)$. PRMT2, arginine N-methyltransferase 2; Dox, doxycycline hyclate.

established to overexpress PRMT2 $\beta$ or PRMT2, and the $p G C-L V-G V 308$ vector was used as a negative control. Stable cell lines either with the PRMT2 $\beta$-3Flag or the PRMT2-3Flag were obtained after selection with puromycin for 2 weeks. Western blot analysis was performed to verify the expression of PRMT2 $\beta$-3Flag or PRMT2-3Flag induced by Dox in the infected MCF7 cells (data not shown). As shown in Fig. 1A, with treatment of $5 \mu \mathrm{g} / \mathrm{ml}$ of Dox, MCF7 cells carrying lentivirus PRMT2 expression exhibited a markedly decreased proliferative ability compared to those without treatment of $5 \mu \mathrm{g} / \mathrm{ml}$ of Dox at the indicated time-points ( $\mathrm{p}<0.05$; Fig. 1A). Whereas, MCF7 cells with lentivirus PRMT2 $\beta$ expression exhibited a weakly decreased proliferative ability compared to those without treatment of $5 \mu \mathrm{g} / \mathrm{ml}$ of Dox (Fig. 1B). The proliferative ability exhibited no difference in the negative control group when treated with or without $5 \mu \mathrm{g} / \mathrm{ml}$ of Dox (data not shown).

Colony formation assay shown in Fig. 1C, with treatment of $5 \mu \mathrm{g} / \mathrm{ml}$ of Dox, revealed that both the number of formed colonies and the cell number of each colony from the MCF7 cells carrying lentivirus PRMT2 expression were markedly decreased compared to the cells without treatment of $5 \mu \mathrm{g} / \mathrm{ml}$ of Dox ( $<<0.01$; Fig. 1C). Whereas, with treatment of Dox, both the number of colonies formed and the cell number of each colony from the MCF7 cells carrying lentivirus PRMT2 $\beta$ expression were weakly decreased compared to the 


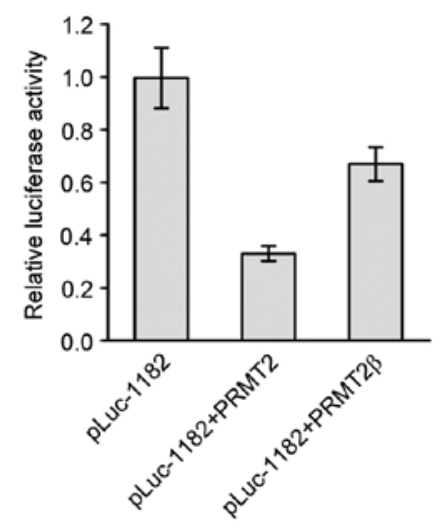

C

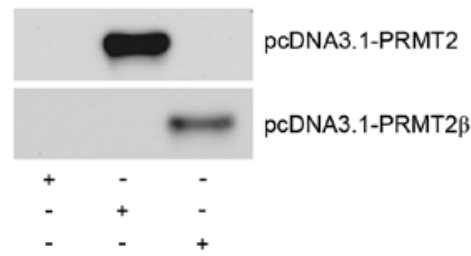

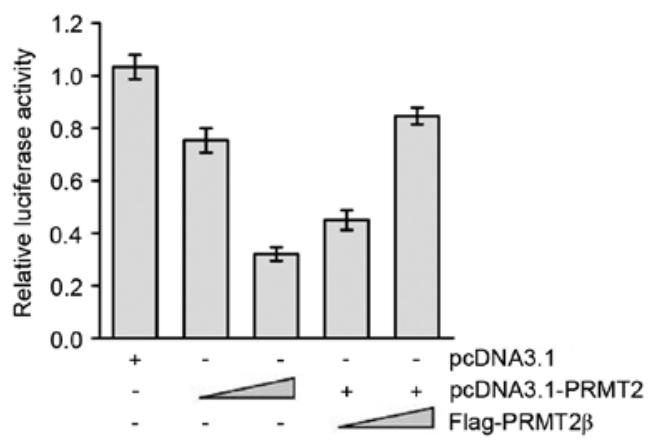

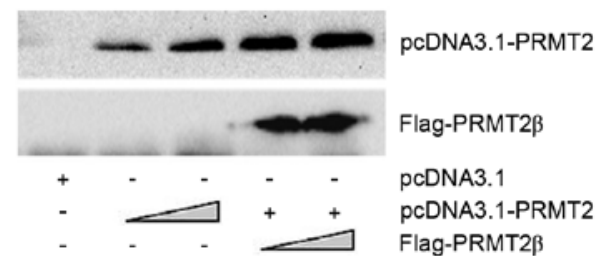

Figure 3. PRMT2 $\beta$ antagonizes the transcription suppressive activity of PRMT2 on the $C C N D 1$ promoter. (A) PRMT2 $\beta$ suppresses the activity of the $C C N D 1$ promoter. $293 \mathrm{~T}$ cells were transfected with indicated constructs together with a CCND1 luciferase reporter plasmid (pLuc-1182). Twenty-four hours after transfection, the cells were harvested, and the luciferase activity was assessed and normalized to Renilla activity. Each bar represents the mean \pm SD for triplicate experiments. (B) The transcription suppression of PRMT2 activity was alleviated by PRMT2 $\beta$. 293T cells were plated in 24 -well plates 1 day before transfection. For transfections, $200 \mathrm{ng}$ of pLuc-1182 reporter plasmid, $100 \mathrm{ng}$ of pcDNA3.1 or 100 ng of pcDNA3.1-PRMT2 constructs plus increasing amounts of pcDNA3.1-PRMT2 $\beta(0,10$, or $100 \mathrm{ng})$ constructs were used. Total plasmid DNAs were made up to $400 \mathrm{ng}$ with pcDNA3.1 empty vectors in each transfection. The cells were collected $24 \mathrm{~h}$ after transfection. The luciferase activity was assessed and normalized to Renilla activity. Each bar represents the mean \pm SD for triplicate experiments. (C) The protein expression of pcDNA3.1-PRMT2 and pcDNA3.1-PRMT2 $\beta$ was confirmed using western blotting with anti-PRMT2 or anti-FLAG. PRMT2, arginine N-methyltransferase 2

cells without treatment of $5 \mu \mathrm{g} / \mathrm{ml}$ of Dox ( $<<0.05$; Fig. 1C). Meanwhile, with soft agar colony formation assay we found that both the number and the size of formed colonies from the MCF7 cells carrying lentivirus PRMT2 $\beta$ expression were significantly decreased compared to the cells without treatment of $5 \mu \mathrm{g} / \mathrm{ml}$ of Dox ( $<<0.05$; Fig. 1D). Similar results were also observed in the cells carrying lentivirus of PRMT2 expression, and the proliferation inhibition effect PRMT2 was shown to be more profound than that of PRMT $2 \beta$ ( $p<0.01$; Fig. 1D). These results indicated that PRMT2 $\beta$ suppressed the cell proliferation and colony formation of breast cancer MCF7 cells.

PRMT2 $\beta$ induces cell cycle arrest and apoptosis of MCF7 cells. We then carried out cell cycle analysis to further characterize the suppressive effects of PRMT2 $\beta$ on the cell cycle. MCF7 cell populations in the $G_{0}-G_{1}$ and $S$ phases were 57.74 and $38.53 \%$ in the PRMT2 $\beta$ (+ Dox) group, while they were 54.15 and $41.65 \%$ in the PRMT2 $\beta$ (- Dox) group. After 3 independent experiments, in the PRMT2 $\beta$ (+ Dox) group, the population of cells in the $\mathrm{G}_{0}-\mathrm{G}_{1}$ phase increased (vs. - Dox, $\mathrm{p}<0.01$; Fig. 2A) and the population of cells in the $\mathrm{S}$ phase significantly decreased (vs. - Dox, $\mathrm{p}<0.01$; Fig. 2A). Moreover, MCF7 cell populations in the $\mathrm{G}_{0}-\mathrm{G}_{1}$ and $\mathrm{S}$ phases were $69.38 \%$ and $30.31 \%$ in the PRMT2 (+ Dox) group, while they were $63.28 \%$ and $35.33 \%$ in the PRMT2 (- Dox) group. After 3 independent experiments, in the PRMT2 (+ Dox) group, the population of cells in the $G_{0}-G_{1}$ phase increased (vs. - Dox, $\mathrm{p}<0.001$; Fig. 2A) and the population of cells in the $\mathrm{S}$ phase significantly decreased (vs. - Dox, p<0.001; Fig. 2A).
These results revealed that PRMT2 $\beta$ significantly induced $\mathrm{G}_{0}-\mathrm{G}_{1}$ phase arrest in MCF7 cells.

We further examined the effect of PRMT2 $\beta$ on apoptosis in MCF7 cells. As shown in Fig. 2B, the percentage of apoptotic cells was $6.89 \%$ in the PRMT2 $\beta$ (+ Dox) group, and $5.5 \%$ in the PRMT2 $\beta$ (- Dox) group. After 3 independent experiments, in the PRMT2 $\beta$ (+ Dox) group, the apoptosis of the MCF7 cells increased (vs. - Dox, p<0.01; Fig. 2B). Furthermore, the percentage of apoptotic MCF7 cells in the PRMT2 (+ Dox) group was $6.39 \%$, and $3.57 \%$ in the PRMT2 (- Dox) group. After 3 independent experiments, in the PRMT2 (+ Dox) group, the apoptosis of the MCF7 cells increased (vs. - Dox, $\mathrm{p}<0.001$; Fig. 2B). These results revealed that PRMT2 $\beta$ induced the apoptosis in MCF7 cells.

PRMT2 $\beta$ antagonizes the transcription suppressive activity of PRMT2 on the CCND1 promoter. In a previous study, we identified that PRMT2 suppresses the CCND1 promoter activity through indirect binding with the AP-1 site in the $C C N D 1$ promoter and functions as a transcription coregulator (15). To understand the involvement of PRMT2 $\beta$ in gene transcription regulation, 293T cells were co-transfected with pcDNA3.1-PRMT2 and/or pcDNA3.1PRMT2 $\beta$ and CCND1 luciferase reporter (pLuc-1182). The results indicated that transfection of either pcDNA3.1-PRMT2 or pcDNA3.1-PRMT2 $\beta$ resulted in the suppression of the reporter activity (Fig. 3A), and the suppressive activity of pcDNA3.1-PRMT2 $\beta$ was weaker compared with that of pcDNA3.1-PRMT2. Co-transfection of FLAG-PRMT2 $\beta$ with 
A

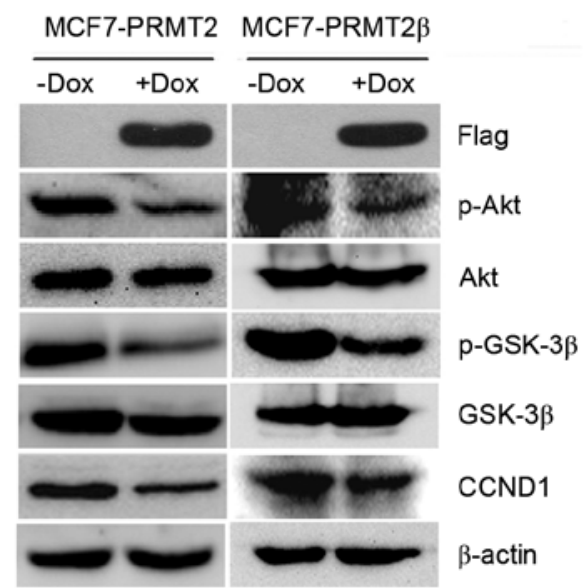

B

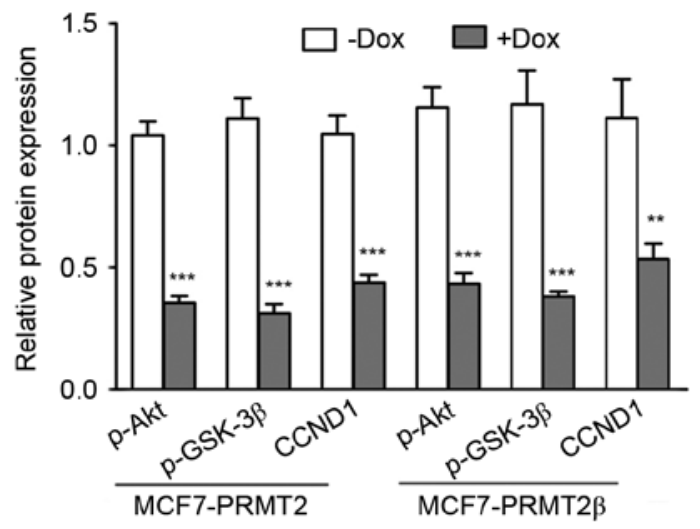

Figure 4. PRMT2 $\beta$ suppresses the expression of CCND1 in breast cancer MCF7 cells. (A) Western blot analysis of MCF7 cells in the - Dox and + Dox groups. Total protein extracts were assessed for total and phosphorylated Akt and GSK3 $\beta$, and CCND1. The expression of p-Akt, p-GSK3 $\beta$ and CCND1 was decreased by PRMT2 $\beta$ and PRMT2 overexpression, respectively. Actin served as a loading control. (B) Relative protein levels of p-Akt, p-GSK3 $\beta$ and CCND1 normalized to $\beta$-actin in MCF7 cells $\left(* * 0<001,{ }^{* * *}\right.$ p $\left.<0.001\right)$. PRMT2, arginine N-methyltransferase 2; Dox, doxycycline hyclate.

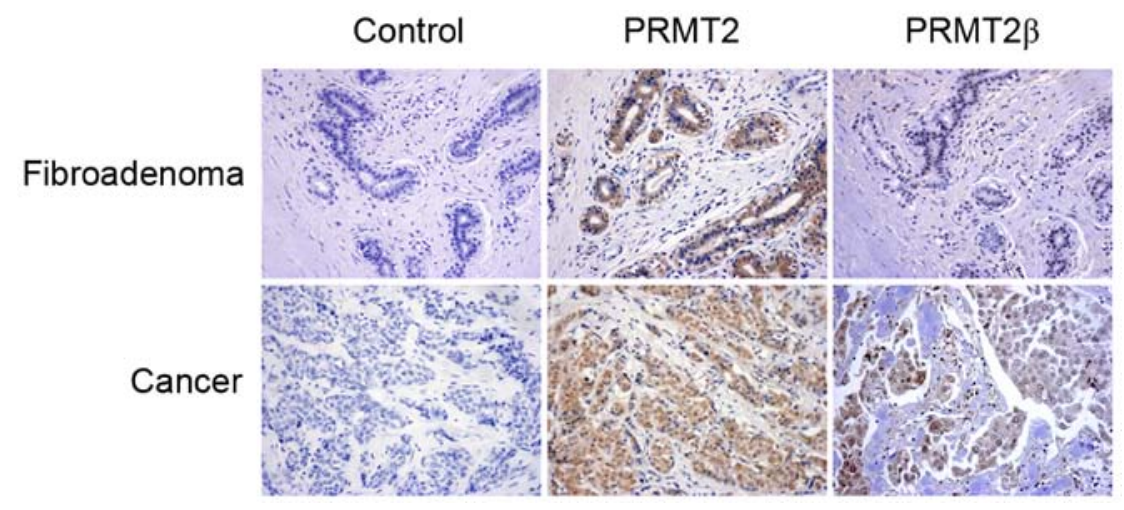

Figure 5. Protein expression of PRMT2 $\beta$ and PRMT2 in breast fibroadenoma and carcinoma tissues (images in original magnification, x200). PRMT2, arginine N-methyltransferase 2 .

pcDNA3.1-PRMT2 led to a relieved suppression of the reporter activity by PRMT2 (Fig. 3B), suggesting that PRMT2 $\beta$ could antagonize the transcription suppression activity of PRMT2. The protein expression of pcDNA3.1-PRMT2, pcDNA3.1PRMT $2 \beta$ and FLAG-PRMT2 $\beta$ was examined by western blotting with anti-PRMT2 and anti-FLAG antibodies (Fig. 3C).

PRMT2 $\beta$ inhibits CCND1 expression via the suppression of Akt/GSK-3 $\beta$ signaling in breast cancer cells. Our previous study reported that PRMT2 suppressed the CCND1 expression in MCF-7 cells partially via the suppression of Akt/GSK-3 $\beta$ signaling. We therefore explored whether PRMT2 $\beta$ was involved in the Akt/GSK-3 $3 /$ CCND1 axis in MCF7 cells. As shown in Fig. 4, with treatment of $5 \mu \mathrm{g} / \mathrm{ml}$ of Dox, the expression of p-Akt and p-GSK3 $\beta$ in MCF7 cells carrying lentivirus PRMT2 $\beta$ expression was markedly decreased compared to the cells without treatment of $5 \mu \mathrm{g} / \mathrm{ml}$ of Dox. Lentivirus-mediated PRMT2 $\beta$ overexpression also markedly suppressed the expression of CCND1, suggesting that both the overexpression of PRMT2 $\beta$ and PRMT2 suppressed Akt/GSK-3 $\beta / C C N D 1$ signaling. These results support the notion that PRMT2 $\beta$ suppressed the CCND1
Table I. Expression of PRMT2 $\beta$ and PRMT2 in normal breast tissue and tumors.

\begin{tabular}{|c|c|c|c|c|c|c|c|}
\hline & \multirow[b]{2}{*}{$\mathrm{n}$} & \multicolumn{2}{|c|}{ PRMT2 } & \multirow[b]{2}{*}{ P-value } & \multicolumn{2}{|c|}{ PRMT2 $\beta$} & \multirow[b]{2}{*}{ P-valu } \\
\hline & & Neg. & Pos. & & Neg. & Pos. & \\
\hline Tissue types & & & & 0.000 & & & 0.096 \\
\hline Normal & 6 & 4 & 2 & & 2 & 4 & \\
\hline Benign & 6 & 1 & 5 & & 3 & 3 & \\
\hline Malignant & 138 & 6 & 132 & & 98 & 40 & \\
\hline
\end{tabular}

Neg., negative; Pos., positive.

expression in MCF-7 cells partially via the suppression of Akt/GSK-3 $\beta$ signaling.

PRMT2 $\beta$ expression is negatively correlated with human epidermal growth factor receptor 2 (HER2). To further identify the association of PRMT2 $\beta$ with breast cancer progression, a tissue microarray (BR1503b; US Biomax, Inc.) consisting of 
Table II. Correlation of PRMT2 $\beta$ and PRMT2 expression with clinicopathological parameters in breast carcinoma.

\begin{tabular}{|c|c|c|c|c|c|c|c|c|c|c|}
\hline & \multirow[b]{2}{*}{$\mathrm{n}$} & \multicolumn{4}{|c|}{ PRMT2 } & \multirow[b]{2}{*}{ P-value } & \multicolumn{3}{|c|}{ PRMT $2 \beta$} & \multirow[b]{2}{*}{ P-value } \\
\hline & & - & + & ++ & +++ & & - & + & ++ & \\
\hline AR & & & & & & 0.641 & & & & 0.430 \\
\hline- & 95 & 5 & 62 & 25 & 3 & & 65 & 25 & 5 & \\
\hline+ & 21 & 1 & 18 & 2 & 0 & & 15 & 6 & 0 & \\
\hline++ & 21 & 0 & 11 & 9 & 1 & & 16 & 4 & 1 & \\
\hline+++ & 1 & 0 & 1 & 0 & 0 & & 1 & 0 & 0 & \\
\hline ER & & & & & & 0.714 & & & & 0.844 \\
\hline- & 91 & 5 & 59 & 22 & 5 & & 65 & 20 & 6 & \\
\hline+ & 11 & 0 & 10 & 1 & 0 & & 8 & 3 & 0 & \\
\hline++ & 10 & 0 & 7 & 3 & 0 & & 8 & 2 & 0 & \\
\hline+++ & 26 & 1 & 15 & 10 & 0 & & 16 & 10 & 0 & \\
\hline PR & & & & & & 0.039 & & & & 0.766 \\
\hline- & 100 & 4 & 72 & 19 & 5 & & 71 & 25 & 4 & \\
\hline+ & 15 & 1 & 8 & 6 & 0 & & 12 & 3 & 0 & \\
\hline++ & 9 & 0 & 4 & 5 & 0 & & 8 & 1 & 0 & \\
\hline+++ & 14 & 0 & 8 & 6 & 0 & & 7 & 5 & 2 & \\
\hline HER2 & & & & & & 0.019 & & & & 0.033 \\
\hline- & 113 & 3 & 82 & 28 & 0 & & 76 & 31 & 6 & \\
\hline+ & 17 & 2 & 7 & 5 & 3 & & 15 & 2 & 0 & \\
\hline++ & 7 & 1 & 1 & 3 & 2 & & 6 & 1 & 0 & \\
\hline+++ & 1 & 0 & 1 & 0 & 0 & & 1 & 0 & 0 & \\
\hline Ki-67 & & & & & & 0.400 & & & & 0.738 \\
\hline- & 83 & 4 & 57 & 19 & 3 & & 59 & 20 & 4 & \\
\hline+ & 19 & 2 & 9 & 8 & 0 & & 14 & 4 & 1 & \\
\hline++ & 24 & 0 & 16 & 6 & 2 & & 19 & 4 & 1 & \\
\hline+++ & 12 & 0 & 9 & 3 & 0 & & 5 & 7 & 0 & \\
\hline p53 & & & & & & 0.648 & & & & 0.296 \\
\hline- & 50 & 2 & 32 & 16 & 0 & & 35 & 13 & 2 & \\
\hline+ & 38 & 1 & 30 & 7 & 0 & & 24 & 12 & 2 & \\
\hline++ & 14 & 1 & 7 & 5 & 1 & & 10 & 2 & 2 & \\
\hline+++ & 36 & 2 & 22 & 8 & 4 & & 29 & 7 & 0 & \\
\hline
\end{tabular}

AR, androgen receptor; ER, estrogen receptor; PR, progesterone receptor; HER2, human epidermal growth factor receptor 2.

138 breast malignant tumor cases, 6 normal cases and 6 benign tumor cases was used. The tissue microarray analysis revealed that the PRMT2 $\beta$ protein expression exhibited a decreased tendency in different tissue types: $66.7 \%$ in normal breast tissue, $50.0 \%$ in breast benign tumors and $29.0 \%$ in breast malignant tumors, although the statistical analysis revealed no significant difference $(\mathrm{p}=0.096$; Table I). Conversely, the percentage of cases with PRMT2 protein expression increased from normal breast tissue to breast malignant tumors: $33.3 \%$ in normal breast tissue, $83.3 \%$ in breast benign tumors and $95.7 \%$ in breast malignant tumors, and the difference was significant ( $\mathrm{p}=0.000$; Table I). The immunostainings of breast fibroadenoma and carcinoma tissue of representative cases are shown in Fig. 5. Furthermore, statistical analysis confirmed that the PRMT2 $\beta$ expression was negatively correlated with HER2 ( $p=0.033$; Table II), when analyzed regardless of breast tumor types. PRMT2 $\beta$ expression in breast carcinoma did not exhibit a statistically significant correlation with androgen receptor (AR), estrogen receptor (ER), progesterone receptor (PR), Ki-67 and the p53 status of the tumor ( $>00.05$; Table II). PRMT2 expression in breast carcinoma also exhibited a statistically significant correlation with the PR and HER2 status ( $\mathrm{p}=0.039$ and 0.019 , respectively; Table II).

\section{Discussion}

Protein arginine methyltransferases (PRMTs) catalyze the methylation of a variety of protein substrates, and there are 9 PRMTs encoded in mammalian genomes. PRMTs are generally ubiquitously expressed and involved in multiple cellular processes including cell growth (16), differentiation (17), embryogenesis (18), nuclear/cytoplasmic protein shuttling (19), RNA splicing and transport $(20,21)$, and post-transcriptional regulation (22). Dysregulation of PRMTs may probably be 
implicated in the pathogenesis of some different diseases, particularly cancer. Accumulating evidence has revealed that overexpression of these enzymes is often associated with various cancers (23-26), which may make some of them viable targets for therapeutic strategies (6). Recently, the existence of alternatively spliced isoforms of PRMTs provides an additional layer of complexity. Specifically, PRMT1, PRMT2, CARM1 and PRMT7 have been shown to have alternative isoforms and most of them play an essential role on the progression and aggressiveness of cancer (24,27-30).

Our previous study found several alternative splicing variants of PRMT2, which are missing different exons in the C-terminals of the wild-type PRMT2 gene, and produce different isoforms. Particularly, due to frame-shifting, PRMT2 $\beta$ loses original structure, and encodes a novel amino acid sequence at the C-terminus of the protein (14). PRMT2 suppressed E2F1 transcriptional activity in an RB-dependent manner (31). PRMT2 also inhibited NF- $\mathrm{KB}$ function and promoted cell apoptosis (11). Our previous study found that suppression of PRMT2 expression promoted the cell proliferation of breast cancer MCF7 cells (15). To explore the role of PRMT2 $\beta$ in the cell proliferation, apoptosis and cell cycle in breast cancer cells, stable cell lines with PRMT2 $\beta-3$ Flag or PRMT2-3Flag were established. We found that the expression of PRMT2 $\beta$ suppressed the cell proliferation and colony formation of MCF7 cells. We also found that PRMT2 $\beta$ expression induced cell cycle arrest and apoptosis of MCF7 cells. In agreement with our previous observations, the wild-type PRMT2 also inhibited breast cancer cell growth. Furthermore, the function of PRMT2 $\beta$ was less efficient than that of the wild-type PRMT2. This may be due to the loss of exons 7-10 in PRMT2 $\beta$, which affects the location of some nuclear receptor-binding interfaces in the $\mathrm{C}$-terminus. However, the mechanism requires further exploration (32).

In a previous study, we identified that PRMT2 suppresses the $C C N D 1$ promoter activity through indirect binding with the AP-1 site in the CCNDI promoter, and functions as a transcription coregulator (15). To understand the cellular function of PRMT2 $\beta$, we further investigated the role of PRMT2 $\beta$ in transcription regulation with $293 \mathrm{~T}$ cells. We found that both PRMT2 $\beta$ and PRMT2 suppressed the activity of the CCND1 promoter, and that the suppressive activity of PRMT2 $\beta$ was weaker compared with that of PRMT2. With coexpression of PRMT2 $\beta$ and PRMT2, the bigger the amount of PRMT2 $\beta$ was, the weaker the inhibition of the CCND1 promoter activity was. We speculated that PRMT2 $\beta$ antagonizes the transcription suppressive activity of PRMT2 by competitively binding to the AP-1 site of the CCND1 promoter, attenuating the transcriptional suppression of PRMT2. It is well known that CCND1 overexpression is a common event in cancer and usually leads to defective modulation at the post-translational level $(33,34)$. Therefore, regulation of the CCND1 protein level is one of the critical aspects in cell proliferation and tumor progression. We had previously demonstrated that miRNA-mediated PRMT2 downregulation promotes CCND1 expression via activation of Akt/GSK-3 $3 /$ CCND1 signaling in breast cancer cells (15). In the present study, we confirmed that lentivirus-mediated PRMT2 $\beta$ expression also significantly decreased Akt/GSK-3 $3 /$ CCND1 signaling, consistent with the results of wild-type PRMT2, indicating that PRMT2 $\beta$ functions as another negative modulator of the Akt/GSK-3 $\beta / C C N D 1$ axis leading to the suppression of proliferation of breast cancer cells.

The tissue microarray analysis revealed that the PRMT2 $\beta$ protein expression exhibited a decreased expression in breast malignant tissue, although the statistical analysis revealed that this difference was not significant, indicating that PRMT2 $\beta$ plays a role in normal breast development and that the loss of PRMT2 $\beta$ expression may be related with the progression of breast cancer, consistent with the results from a cell model. Further analysis of the tissue microarray data revealed that PRMT $2 \beta$ expression was negatively correlated with HER2 $(\mathrm{p}=0.033)$, although the molecular mechanism remains unknown. Further investigations are required to clarify the alternative molecular mechanisms of PRMT2 $\beta$ in the progression of breast cancer. In summary, our results revealed that PRMT2 $\beta$, a novel splice variant of PRMT2, has a potential antitumor effect through the suppression of CCND1 expression and inhibition of Akt signaling activity. This also opens a new avenue for treating breast cancer.

\section{Acknowledgements}

The present study was supported by projects from the National Natural Science Foundation of China (grant nos. 31200573, 81272355 and 81472608), the Key Project of the Education Department of Hunan Province (16A189), the Natural Science Foundation of Hunan Province (2016JJ4077) and the Young Talents Program of the University of South China.

\section{References}

1. Modrek B and Lee C: A genomic view of alternative splicing. Nat Genet 30: 13-19, 2002.

2. Johnson JM, Castle J, Garrett-Engele P, Kan Z, Loerch PM, Armour CD, Santos R, Schadt EE, Stoughton R and Shoemaker DD: Genome-wide survey of human alternative pre-mRNA splicing with exon junction microarrays. Science 302: 2141-2144, 2003.

3. Resch A, Xing Y, Modrek B, Gorlick M, Riley R and Lee C: Assessing the impact of alternative splicing on domain interactions in the human proteome. J Proteome Res 3: 76-83, 2004.

4. Sorek R, Shamir R and Ast G: How prevalent is functional alternative splicing in the human genome? Trends Genet 20: 68-71, 2004.

5. Baldwin RM, Morettin A and Côté J: Role of PRMTs in cancer: Could minor isoforms be leaving a mark? World J Biol Chem 5: 115-129, 2014.

6. Yang Y and Bedford MT: Protein arginine methyltransferases and cancer. Nat Rev Cancer 13: 37-50, 2013.

7. Herrmann F, Pably P, Eckerich C, Bedford MT and Fackelmayer FO: Human protein arginine methyltransferases in vivo - distinct properties of eight canonical members of the PRMT family. J Cell Sci 122: 667-677, 2009.

8. Lakowski TM and Frankel A: Kinetic analysis of human protein arginine N-methyltransferase 2: Formation of monomethyl- and asymmetric dimethyl-arginine residues on histone $\mathrm{H} 4$. Biochem J 421: 253-261, 2009.

9. Besson V, Brault V, Duchon A, Togbe D, Bizot JC, Quesniaux VF, Ryffel B and Hérault Y: Modeling the monosomy for the telomeric part of human chromosome 21 reveals haploinsufficient genes modulating the inflammatory and airway responses. Hum Mol Genet 16: 2040-2052, 2007

10. Blythe SA, Cha SW, Tadjuidje E, Heasman J and Klein PS: beta-Catenin primes organizer gene expression by recruiting a histone $\mathrm{H} 3$ arginine 8 methyltransferase, Prmt2. Dev Cell 19: 220-231, 2010.

11. Ganesh L, Yoshimoto T, Moorthy NC, Akahata W, Boehm M, Nabel EG and Nabel GJ: Protein methyltransferase 2 inhibits NF-kappaB function and promotes apoptosis. Mol Cell Biol 26: 3864-3874, 2006. 
12. Iwasaki H, Kovacic JC, Olive M, Beers JK, Yoshimoto T, Crook MF, Tonelli LH and Nabel EG: Disruption of protein arginine $N$-methyltransferase 2 regulates leptin signaling and produces leanness in vivo through loss of STAT3 methylation. Circ Res 107: 992-1001, 2010.

13. Yildirim AO, Bulau P, Zakrzewicz D, Kitowska KE, Weissmann N, Grimminger F, Morty RE and Eickelberg O Increased protein arginine methylation in chronic hypoxia: Role of protein arginine methyltransferases. Am J Respir Cell Mol Biol 35: 436-443, 2006.

14. Zhong J, Cao RX, Zu XY, Hong T, Yang J, Liu L, Xiao XH, Ding WJ, Zhao Q, Liu JH, et al: Identification and characterization of novel spliced variants of PRMT2 in breast carcinoma. FEBS J 279: 316-335, 2012

15. Zhong J, Cao RX, Liu JH, Liu YB, Wang J, Liu LP, Chen YJ, Yang J, Zhang QH, Wu Y, et al: Nuclear loss of protein arginine $\mathrm{N}$-methyltransferase 2 in breast carcinoma is associated with tumor grade and overexpression of cyclin D1 protein. Oncogene 33: 5546-5558, 2014.

16. Lin WJ, Gary JD, Yang MC, Clarke S and Herschman HR: The mammalian immediate-early TIS 21 protein and the leukemia-associated BTG1 protein interact with a proteinarginine $N$-methyltransferase. J Biol Chem 271: 15034-15044, 1996.

17. Chen SL, Loffler KA, Chen D, Stallcup MR and Muscat GEO: The coactivator-associated arginine methyltransferase is necessary for muscle differentiation: CARM1 coactivates myocyte enhancer factor-2. J Biol Chem 277: 4324-4333, 2002.

18. Torres-Padilla ME, Parfitt DE, Kouzarides T and Zernicka-Goetz M: Histone arginine methylation regulates pluripotency in the early mouse embryo. Nature 445: 214-218, 2007.

19. McBride AE and Silver PA: State of the arg: Protein methylation at arginine comes of age. Cell 106: 5-8, 2001.

20. Lukong KE and Richard S: Arginine methylation signals mRNA export. Nat Struct Mol Biol 11: 914-915, 2004.

21. Meister G and Fischer U: Assisted RNP assembly: SMN and PRMT5 complexes cooperate in the formation of spliceosomal UsnRNPs. EMBO J 21: 5853-5863, 2002.

22. Li H, Park S, Kilburn B, Jelinek MA, Henschen-Edman A, Aswad DW, Stallcup MR and Laird-Offringa IA: Lipopolysaccharide-induced methylation of HuR, an mRNA-stabilizing protein, by CARM1. Coactivator-associated arginine methyltransferase. J Biol Chem 277: 44623-44630, 2002.

23. Zhang B, Dong S, Li Z, Lu L, Zhang S, Chen X, Cen X and $\mathrm{Wu}$ Y: Targeting protein arginine methyltransferase 5 inhibits human hepatocellular carcinoma growth via the downregulation of beta-catenin. J Transl Med 13: 349, 2015.
24. Goulet I, Gauvin G, Boisvenue S and Côté J: Alternative splicing yields protein arginine methyltransferase 1 isoforms with distinct activity, substrate specificity, and subcellular localization. J Biol Chem 282: 33009-33021, 2007

25. El Messaoudi S, Fabbrizio E, Rodriguez C, Chuchana P, Fauquier L, Cheng D, Theillet C, Vandel L, Bedford MT and Sardet C: Coactivator-associated arginine methyltransferase 1 (CARM1) is a positive regulator of the Cyclin E1 gene. Proc Natl Acad Sci USA 103: 13351-13356, 2006.

26. Thomassen M, Tan Q and Kruse TA: Gene expression metaanalysis identifies chromosomal regions and candidate genes involved in breast cancer metastasis. Breast Cancer Res Treat 113: 239-249, 2009.

27. Baldwin RM, Morettin A, Paris G, Goulet I and Côté J: Alternatively spliced protein arginine methyltransferase 1 isoform PRMT1v2 promotes the survival and invasiveness of breast cancer cells. Cell Cycle 11: 4597-4612, 2012.

28. Zhong J, Cao RX, Hong T, Yang J, Zu XY, Xiao XH, Liu JH and Wen GB: Identification and expression analysis of a novel transcript of the human PRMT2 gene resulted from alternative polyadenylation in breast cancer. Gene 487: 1-9, 2011.

29. Ohkura N, Takahashi M, Yaguchi H, Nagamura Y and Tsukada T: Coactivator-associated arginine methyltransferase 1, CARM1, affects pre-mRNA splicing in an isoform-specific manner. J Biol Chem 280: 28927-28935, 2005.

30. Gros L, Renodon-Cornière A, de Saint Vincent BR, Feder M, Bujnicki JM and Jacquemin-Sablon A: Characterization of prmt7alpha and beta isozymes from Chinese hamster cells sensitive and resistant to topoisomerase II inhibitors. Biochim Biophys Acta 1760: 1646-1656, 2006.

31. Yoshimoto T, Boehm M, Olive M, Crook MF, San H, Langenickel $\mathrm{T}$ and Nabel EG: The arginine methyltransferase PRMT2 binds RB and regulates E2F function. Exp Cell Res 312: 2040-2053, 2006.

32. Meyer R, Wolf SS and Obendorf M: PRMT2, a member of the protein arginine methyltransferase family, is a coactivator of the androgen receptor. J Steroid Biochem Mol Biol 107: 1-14, 2007.

33. Tashiro E, Tsuchiya A and Imoto M: Functions of cyclin D1 as an oncogene and regulation of cyclin D1 expression. Cancer Sci 98: 629-635, 2007.

34. Kim JK and Diehl JA: Nuclear cyclin D1: An oncogenic driver in human cancer. J Cell Physiol 220: 292-296, 2009. 